\title{
ESTUDIO COMPARATIVO DE LOS DEFECTOS DE REFRACCIÓN EN NIÑOS CON PTOSIS MIOGÉNICA CONGÉNITA SIMPLE Y NIÑOS CONTROL
}

\section{COMPARATIVE STUDY OF REFRACTIVE ERRORS IN SIMPLE CONGENITAL MYOGENIC PTOSIS AND CONTROL CHILDREN}

\author{
PÉREZ-ÍÑIGO MA ${ }^{1}$, GONZÁLEZ I ${ }^{1}$, MAYORAL $F^{1}$, FERRER $C^{2}$, HONRUBIA FM ${ }^{3}$
}

\section{RESUMEN}

Propósito: Estudiar los defectos de refracción en niños con ptosis miogénica congénita simple de diferentes grados y realizar un estudio comparativo respecto a niños control en nuestra población.

Método: Se han incluido 35 niños con ptosis miogénica congénita simple, de los que 27 tenían ptosis leve, 3 ptosis moderada y 5 ptosis grave, y 35 niños control. Se ha incluido un ojo de cada paciente, el peor en caso de ptosis, y aleatorio en caso de niño control. Se ha realizado un estudio comparativo de los datos refractivos obtenidos en cada población, mediante los test estadísticos: $t$ de student, ANOVA y Chi cuadrado o Chi cuadrado con corrección de Yates.

Resultados: Se han obtenido diferencias significativas entre niños con ptosis miogénica congénita simple y niños control en equivalente esférico medio $(3,08 / 1,49)$, en esfera absoluta media $(2,80 / 1,42)$ y en cilindro absoluto medio $(0,81 / 0,31)$ $(\mathrm{p}<0,05)$.

Se ha observado una asociación entre la existencia de astigmatismo y la gravedad de la ptosis $\left(\chi^{2}=6,88>5,99\right)$ y entre la necesidad de corrección óptica y la existencia de ptosis $\left(\chi^{2}=15,92>3,84\right)$.

Conclusiones: Los niños con ptosis miogénica congénita simple de nuestro medio tienen más defectos

\section{ABSTRACT}

Purpose: To study refractive errors in children with relatively serious congenital myogenic ptosis and to carry out a comparative study in relation to control children in our population.

Methods: We included 35 children with simple congenital myogenic ptosis, 27 of them had minor ptosis, 3 moderate ptosis and 5 serious ptosis. Thirty-five children were also in the control group. One eye of every subject was included, the worst eye in subjects of the ptosis group and an eye selected at random in control group subjects.

A comparative study of refractive data in every group was carried out, using the Student's t-test, ANOVA and Chi square or Yates correction tests.

Results: We obtained significant differences between children with ptosis and controls in average spherical equivalent (3.08/1.49), in average absolute sphere (2.80/1.42) and in average absolute cylin$\operatorname{der}(0.81 / 0.31)(\mathrm{p}<0.05)$.

We also observed a relation between the presence of astigmatism and the seriousness of ptosis $\left(\chi^{2}=6.88>5.99\right)$, and between the need for optical correction and the presence of ptosis $\left(\chi^{2}=15.92>3.84\right)$. Conclusions: Children with simple congenital myogenic ptosis in our enviroment have greater refracti-

\footnotetext{
Recibido: 31/5/06. Aceptado: 26/9/08.

1 Doctor en Medicina. Hospital Universitario Miguel Servet. Zaragoza. España.

2 Licenciada en Medicina. Hospital Universitario Miguel Servet. Zaragoza. España.

3 Doctor en Medicina. Catedrático de Oftalmología. Facultad de Medicina. Universidad de Zaragoza. Zaragoza. España.
}

Correspondencia:

M. a Aránzazu Pérez Íñigo

Servicio de Oftalmología. Hospital Universitario Miguel Servet

Isabel la Católica, 1-3

50009 Zaragoza

España

E-mail: aperezinigo@hotmail.com 
de refracción tanto esférico como cilíndrico que los niños control. Los niños con ptosis necesitan corrección óptica con mayor frecuencia que los niños control.

A mayor gravedad de ptosis, mayor es la posibilidad de tener astigmatismo.

Palabras clave: Ptosis miogénica congénita, niños, astigmatismo, defectos de refracción, corrección óptica. ve errors than control children. Ptosis children require optical correction more frequently than control. The more serious the ptosis, the more likelihood there is of having astigmatism (Arch Soc Esp Oftalmol 2008; 83: 601-606).

Key words: Myogenic congenital ptosis, children, astigmatism, refractive errors, optical correction.

\section{INTRODUCCIÓN}

La ptosis es la posición anormalmente baja del párpado superior. Esta puede ser: miogénica, neurogénica, mecánica, aponeurótica y traumática (1).

Ptosis miogénica: Causada por miopatía del músculo elevador o por afectación de la transmisión de impulsos en la unión neuromuscular. Puede ser congénita o adquirida. La adquirida se observa en la miastenia gravis, la distrofia miotónica y las miopatías oculares (2). La congénita consta de la forma simple, que es la más frecuente, la asociada a debilidad del músculo recto superior, la incluida en el síndrome de blefarofimosis y la incluida en el síndrome de fibrosis congénita.

La ptosis miogénica congénita simple se debe a una disgenesia del elevador del párpado superior. Existe una alteración en la contracción para elevar el párpado en la mirada hacia arriba y un déficit de relajación del músculo en la mirada hacia abajo. Cuanto menos desarrollado está el músculo, más grave es la ptosis. Se manifiesta desde el nacimiento, y se mantiene constante toda la vida.

El $75 \%$ de ptosis congénitas son unilaterales y puras. Es raro que exista ambliopía debida únicamente a la oclusión pupilar, generalmente suele ser secundaria a estrabismo convergente, alto astigmatismo o anisometropía $(1,3)$.

El $25 \%$ restante son bilaterales y a veces asimétricas.

La posibilidad de ambliopía y defectos refractivos asociados obligan a su detección precoz y al tratamiento quirúrgico cuando esté indicado. Si la ptosis es muy acusada y provoca ambliopía se debe reparar lo antes posible.

Las técnicas de suspensión del frontal y la resección del elevador son las más utilizadas en los niños porque proporcionan buenos resultados funcionales con los mínimos riesgos quirúrgicos. Cuando se realiza la técnica de suspensión del frontal, el uso de fascia lata es mejor que otros materiales $(4,5)$.

El objetivo de este estudio es comparar los datos refractivos de niños con ptosis miogénica congénita simple de distinta gravedad y niños control.

\section{SUJETOS, MATERIAL Y MÉTODOS}

\section{Selección de la muestra}

Se incluyeron un total de 70 ojos, pertenecientes a 70 niños remitidos a las consultas de oftalmología pediátrica de nuestro centro.

35 de ellos padecían ptosis congénitas simples de gravedad variable, y fueron seleccionados de manera aleatoria a partir de un listado de niños con esta patología. Los otros 35 niños fueron remitidos a nuestras consultas o por lagrimeo al nacimiento o por vía urgencias por patologías banales, y se les hizo un estudio refractivo completo a todos ellos en torno a los 3 - 4 años de edad, que se utilizaron para recoger los datos.

Se seleccionó el peor ojo en los niños con ptosis, y derecho/izquierdo de forma aleatoria en los sujetos control.

Se realizó una anamnesis detallada, exploración oftalmológica completa y estudio de refracción bajo cicloplejía. A los niños con ptosis se les realizó una exploración específica de dicha patología.

\section{Datos demográficos}

Sexo, edad en meses en primera visita y quién los remitía a nuestras consultas (pediatra, oftalmólogo de zona u otros). 


\section{Datos de la ptosis}

Ojo afecto (derecho, izquierdo o ambos), gravedad de la ptosis (leve, moderada o grave), necesidad de cirugía, técnica empleada y complicaciones si existieron.

\section{Definimos}

- Ptosis leve: $2 \mathrm{~mm}$ por debajo de la posición normal del párpado, a nivel del borde pupilar superior.

- Ptosis moderada: 3-4 mm por debajo de la posición normal del párpado, pupila parcialmente cubierta.

- Ptosis severa: Más de 4 mm por debajo, con pupila totalmente cubierta (6).

\section{Datos refractivos}

Agudeza visual, valor del equivalente esférico, valor absoluto de la esfera, presencia de astigmatismo, valor absoluto del cilindro, tipo de cilindro (directo $=+90^{\circ}$ con desviación estándar (DE) $10^{\circ}$ ó $-180^{\circ} \mathrm{DE} 10^{\circ}$, indirecto $=+180^{\circ} \mathrm{DE} 10^{\circ} \mathrm{o}-90^{\circ} \mathrm{DE}$ $10^{\circ}, \mathrm{u}$ oblicuo $=$ resto de casos), necesidad de corrección óptica ( igual o mayor que +3 dioptrías o igual o menor que $-1,5$ dioptrías de esfera, valor absoluto del cilindro y/o anisometropía igual o mayor que 1,5 dioptrías) y presencia de ambliopía (2 o más líneas de diferencia entre ambos ojos).

\section{Estadística}

Para representar gráficamente las frecuencias de las variables nominales se han utilizado diagramas «pastel».

Para comparar los datos cuantitativos entre más de 2 grupos se empleó el test ANOVA.

Para comparar los datos cuantitativos entre 2 grupos se empleó el test t de Student.
Para estudiar la asociación entre variables nominales se utilizaron tablas de contingencia, el estadístico $\chi^{2}$ y $\chi^{2}$ con corrección de Yates en los casos indicados.

Se han considerado significativas las diferencias encontradas con un nivel de significación $\mathrm{p}<0,05$.

\section{RESULTADOS}

Se evaluaron un total de 70 ojos, de los cuales 35 tenían ptosis asociada y 35 ojos de niños control. No se encontraron diferencias significativas en edad media ni en agudeza visual entre ambos grupos (tabla I).

En el grupo con ptosis, había un total de 13 niñas $(37,1 \%)$ y 22 niños $(62,9 \%) .14(40 \%)$ tenían afectado el ojo derecho y $21(60 \%)$ el izquierdo. Un $8,6 \%$ fueron remitidos por el pediatra, un $68,6 \%$ por el oftalmólogo de zona y un $22,9 \%$ por otras vías. Del total de casos, un $77,1 \%$ (27 casos) eran formas leves, un 8,6\% (tres casos) formas moderadas y un $14,3 \%$ (cinco casos) formas severas.

Se llevaron a cabo cinco intervenciones quirúrgicas. En todos los casos se realizó una suspensión del frontal. Hubo complicaciones en dos de los casos, uno de ellos una ptosis leve residual y el otro un absceso localizado que se resolvió sin secuelas.

Con respecto a los datos refractivos, encontramos astigmatismo en 21 casos (60\%), siendo en siete casos $(20 \%)$ directo, en cinco casos $(14,3 \%)$ indirecto y oblicuo en nueve casos $(25,7 \%)$. Necesitaron gafa 21 casos $(60 \%)$ y se clasificaron como ambliopías cinco casos (14,3\%), teniendo 28 casos $(80 \%)$ agudeza visual normal y dos casos $(5,7 \%)$ difíciles de evaluar por edad $<1$ año (fig. 1). Los pacientes con ambliopía tenían una edad media de $1 .^{\mathrm{a}}$ visita de 39 meses. Estos niños tenían agudezas visuales de 0,4 en un caso, 0,6 en tres casos y 0,8 en otro caso. De los cinco niños con ambliopía, tres tenían ptosis leve, uno moderada y uno severa. El resto de casos de ptosis tenían agudezas de 0,8, 0,9 y 1 . La agudeza visual media de los niños con ptosis fue de 0,91.

Del total de niños con ptosis, cinco de ellos tenían ptosis severa, de los cuales cuatro tuvieron una agu-

Tabla I.

\begin{tabular}{lccccccc}
\hline Grupo & $\mathrm{N}$ & AV media & Desviación estándar & $\mathrm{p}$ & Edad media meses 1. ${ }^{\text {a visita }}$ & Desviación estándar & $\mathrm{p}$ \\
\hline Control & 35 & 0,98 & 0,10 & 0,05 & 42,97 & 10,06 & 0,10 \\
Ptosis & 33 & 0,91 & 0,15 & 0.05 & 34,14 & 29,79 & 0,10 \\
\hline \hline
\end{tabular}

N: Número de niños en cada grupo; AV: Agudeza visual; p: Nivel de significación del test t de Student. 

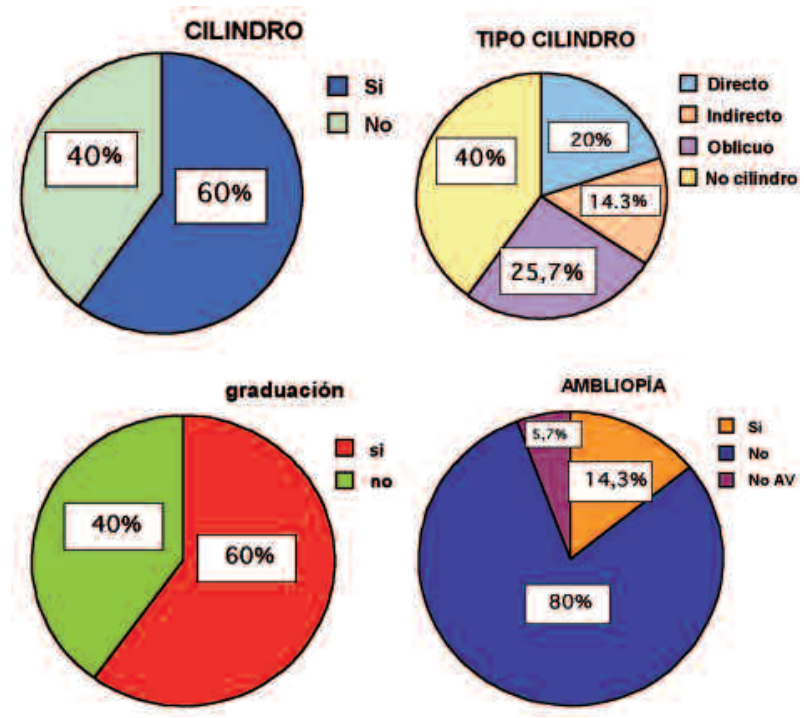

Fig. 1: Distribución de frecuencias en grupo de ptosis de los datos refractivos.

deza de 1 y 1 de 0,8 (único con ambliopía en este subgrupo).

De los pacientes con ptosis severa, fueron intervenidos quirúrgicamente los cinco pacientes, y se les aplicó corrección óptica a cuatro de ellos. En este grupo la edad media de 1. ${ }^{\text {a }}$ visita fue de 22 meses.

En el grupo control, había un total de 19 niñas $(54,3 \%)$ y 16 niños $(45,7 \%) .18$ eran ojos derechos $(51,4 \%)$ y $17(48,6 \%)$ izquierdos. Un $31,4 \%$ fueron remitidos por el pediatra, un 5,7\% por el oftalmólogo de zona y un $62,9 \%$ por otras vías.

Con respecto a los datos refractivos, encontramos astigmatismo en 13 niños $(37,1 \%)$, siendo en ocho casos $(22,9 \%)$ directo, en cuatro casos $(11,4 \%)$ indirecto y en un caso $(2,9 \%)$ oblicuo. Necesitaron gafa en cuatro casos $(11,4 \%)$ y no hubo ningún caso de ambliopía (fig. 2).

Se encontraron diferencias estadísticamente significativas $(\mathrm{p}<0,05)$ entre ambos grupos en la media del equivalente esférico: control $=+1,49$; ptosis $=$ $+3,08$, en la media de esfera: control $=1,42$; ptosis $=2,80$ y en el cilindro absoluto medio: control $=$ 0,31 ; ptosis $=0,81$.

No se encontraron diferencias estadísticamente significativas entre los tipos de ptosis según su gravedad ni en equivalente esférico, ni en esfera, ni en cilindro absoluto (tabla II).

En cuanto a las asociaciones existentes entre las distintas condiciones, se encontró asociación entre la presencia de astigmatismo y la gravedad de ptosis, así como entre la presencia de ptosis y la necesidad de corrección óptica (tablas III y IV).

No se encontró asociación entre la presencia de astigmatismo y la presencia de ptosis, ni entre la presencia de ptosis y el tipo de astigmatismo, ni entre la gravedad de la ptosis y el tipo de astigmatismo ni entre la necesidad de corrección óptica y la gravedad de ptosis, ni entre la presencia de ambliopía y la presencia de ptosis o la presencia de ambliopía y la gravedad de la ptosis.

\section{DISCUSIÓN}

Los niños con ptosis miogénica congénita simple de nuestro medio, tienen más dioptrías de esfera y cilindro que los niños control, y necesitan corrección óptica con mayor frecuencia. Merriam W. ya observó esta circunstancia al comparar la refracción del ojo con ptosis con el contralateral $(3,7,8)$.
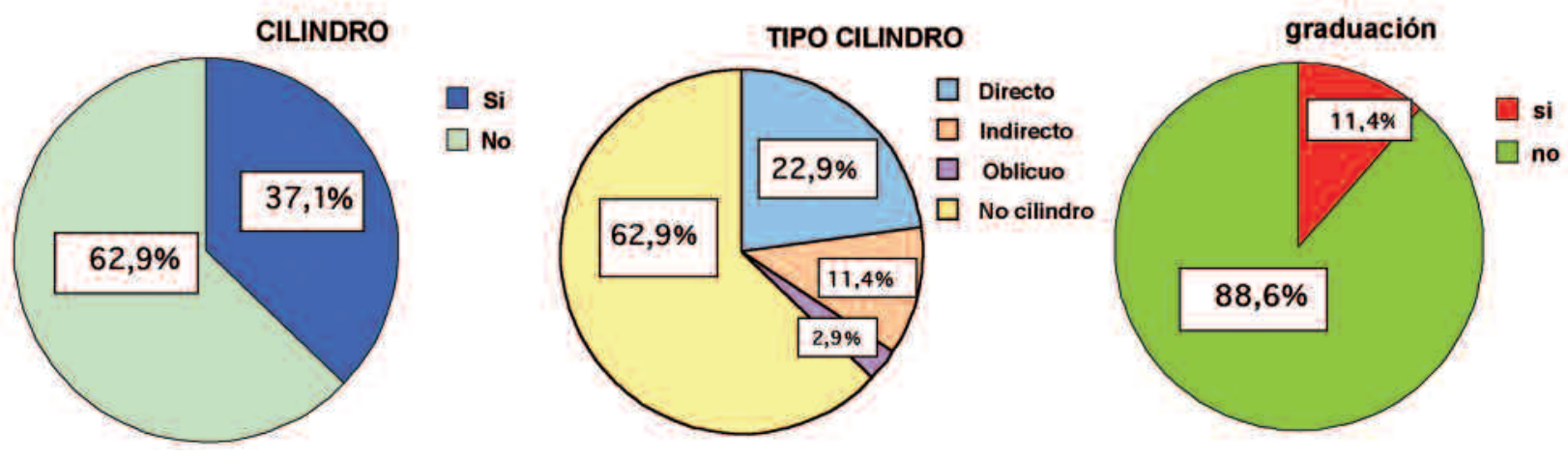

Fig. 2: Distribución de frecuencias en grupo control de los datos refractivos. 
Tabla II.

\begin{tabular}{lcccccccccc}
\hline Grupo & $\mathrm{N}$ & $\begin{array}{c}\text { Equivalente esférico } \\
\text { medio }\end{array}$ & $\mathrm{DE}$ & $\mathrm{p}$ & $\begin{array}{c}\text { Esfera absoluta } \\
\text { media }\end{array}$ & $\mathrm{DE}$ & $\mathrm{p}$ & $\begin{array}{c}\text { Cilindro absoluto } \\
\text { medio }\end{array}$ & $\mathrm{DE}$ & $\mathrm{p}$ \\
\hline Control & 35 & 1,49 & 1,35 & 0,000 & 1,42 & 1,32 & 0,001 & 0,31 & 0,58 & 0,018 \\
Ptosis & 35 & 3,08 & 1,93 & 0,000 & 2,80 & 1,80 & 0,001 & 0,81 & 0,18 & 0,018 \\
\hline \hline
\end{tabular}

N: Número de niños en cada grupo; DE: Desviación estándar; P: Nivel de significación del test t de Student.

Tabla III.

\begin{tabular}{|c|c|c|c|c|}
\hline & Ptosis leve & Ptosis moderada & Ptosis grave & Total \\
\hline Astigmatismo sí & 13 & 3 & 5 & 21 \\
\hline Astigmatismo no & 14 & 0 & 0 & 14 \\
\hline Total & 27 & 3 & 5 & 35 \\
\hline
\end{tabular}

$\chi^{2}=6,88>5,99$.

Además de estudiar un grupo de niños control, se podría comparar con el estado refractivo del ojo adelfo y evaluar las posibles diferencias.

En nuestro grupo, a mayor gravedad de ptosis, mayor es la posibilidad de tener astigmatismo.

Es probable que el bajo número de casos de ptosis moderada y grave, sea el motivo de que no haya asociación entre la gravedad de ptosis y ambliopía o que no se hayan encontrado diferencias en dioptrías según la gravedad. Es posible que la precoz actuación en los casos de ptosis severa fuera fundamental para evitar una evolución hacia la ambliopía.

En los casos de ambliopía la detección y corrección de la ametropía correspondiente fue algo más tardía (edad media 39 meses).

Es curioso que el tipo de astigmatismo es indiferente en nuestro medio de la existencia de ptosis y de la gravedad de la misma.

Tampoco hemos encontrado asociación entre la presencia de ptosis y la presencia de ambliopía, quizás porque la ambliopía es debida muchas veces a otros factores asociados (1). La presencia de ptosis se acompañó de una mayor necesidad de corrección óptica y la gravedad de la misma, de una mayor presencia de astigmatismo, pero con la corrección ade-

\section{Tabla IV.}

Corrección óptica sí Corrección óptica no Total

\begin{tabular}{lcll}
\hline Ptosis sí & 21 & 14 & 35 \\
Ptosis no & 4 & 31 & 35 \\
Total & 25 & 45 & 70 \\
\hline \hline
\end{tabular}

$\chi^{2}=15,92>3,84$. cuada no hubo diferencias significativas en agudeza visual con la población control. Quizás la deficiente estimulación macular en el ojo con ptosis puede producir una mayor ametropización cilíndrica en comparación con la población control. Es posible que la inexistencia de una anisometropía significativa entre el ojo con ptosis y el contralateral explique en parte la ausencia de más ambliopías en este grupo, para comprobarlo deberíamos hacer un estudio comparativo con el ojo adelfo.

La ptosis es una patología relativamente frecuente en el niño y de gran relevancia. Es importante su correcta valoración y la evaluación precoz y adecuada de los defectos de refracción asociados y su corrección así como la indicación de cirugía en los casos necesarios. La técnica utilizada en nuestro medio con más frecuencia es la suspensión del frontal porque proporciona buenos resultados funcionales y pocos riesgos (4).

Nosotros no hemos estudiado la variación de los defectos de refracción tras intervención quirúrgica debido al bajo número de casos, pero sobre este tema sí se ha escrito, y a veces se sugiere un mayor desarrollo del astigmatismo y ambliopía en algunos pacientes operados en los primeros años de vida $(3,9)$.

\section{BIBLIOGRAFÍA}

1. Mateos Sánchez, E, Corcóstegui Cortina J, Alonso Formento MN. Tipos de ptosis palpebral. In: Mateos Sánchez E; Ptosis palpebral. Majadahonda (Madrid): Ergon; 2005; 33-99.

2. Wong VA, Beckingsale PS, Oley CA, Sullivan TJ. Manegement of myogenic ptosis. Ophthtalmology 2002; 109: 1023-1031. 
3. Merriam WW, Ellis FD, Helveston EM. Congenital blepharoptosis, anisometropia, and amblyopia. Am J Ophthalmol 1980; 89: 401-407.

4. Leibovitch I, Leibovitch L, Dray JP. Long-term results of frontalis suspension using autogenous fascia lata for congenital ptosis in children under 3 years of age. Am J Ophthalomol 2003; 136: 866-871.

5. Junceda-Moreno J, Suárez-Suárez E, Dos-Santos-Bernardo $V$. Estudio comparativo de diferentes materiales empleados en la suspensión al frontal en casos de ptosis palpebral. Arch Soc Esp Oftalmol 2005; 80: 457462.
6. Finsterer J. Ptosis: causes, presentation, and management. Aesthetic Plast Surg 2003; 27: 193-204.

7. Stark N, Walther C. Refractive errors, amblyopia and strabismus in congenital ptosis. Klin Monatsbl Augenheilkd 1984; 184: 37-39.

8. Gusek-Schneider GC. Congenital ptosis: amblyogenic refractive errors, amblyopia, manifest strabismus and stereopsis related to the types of ptosis. Data on 77 patients and review of the literature. Klin Monatsbl Augenheilkd 2002; 219: 340-348.

9. Kao SC, Tsai CC, Lee SM, Liu JH. Astigmatic change following congenital ptosis surgery. Zhonghua Yi Xue ZaZhi (Taipei) 1998; 61: 689-693. 